\title{
Harmful Intrusion Detection Algorithm of Optical Fiber Pre-Warning System Based on Correlation of Orthogonal Polarization Signals
}

\author{
Fukun $\mathrm{BI}^{1}$, Chong $\mathrm{FENG}^{1}$, Hongquan $\mathrm{QU}^{1 *}$, Tong $\mathrm{ZHENG}^{1}$, and Chonglei $\mathrm{WANG}^{2}$ \\ ${ }^{1}$ School of Electrical and Information Engineering, North China University of Technology, Beijing, 100144, China \\ ${ }^{2}$ Beijing Institute of Technology Department of Information and Electronic, Beijing, 100081, China \\ ${ }^{*}$ Corresponding author: Hongquan QU？ E-mail: dolly115dolly@163.com
}

\begin{abstract}
At present, advanced researches of optical fiber intrusion measurement are based on the constant false alarm rate (CFAR) algorithm. Although these conventional methods overcome the interference of non-stationary random signals, there are still a large number of false alarms in practical applications. This is because there is no specific study on orthogonal polarization signals of false alarm and intrusion. In order to further reduce false alarms, we analyze the correlation of optical fiber signals using birefringence of single-mode fiber. This paper proposes the harmful intrusion detection algorithm based on the correlation of two orthogonal polarization signals. The proposed method uses correlation coefficient to distinguish false alarms and intrusions, which can decrease false alarms. Experiments on real data, which are collected from the practical environment, demonstrate that the difference in correlation is a robust feature. Furthermore, the results show that the proposed algorithm can reduce the false alarms and ensure the detection performance when it is used in optical fiber pre-warning system (OFPS).
\end{abstract}

Keywords: Optical fiber; birefringence; orthogonal polarization signals; correlation

Citation: Fukun BI, Chong FENG, Hongquan QU, Tong ZHENG, and Chonglei WANG, "Harmful Intrusion Detection Algorithm of Optical Fiber Pre-Warning System Based on Correlation of Orthogonal Polarization Signals," Photonic Sensors, 2017, 7(3): 226-233.

\section{Introduction}

The optical fiber pre-warning system (OFPS) can be extensively applied in the fields of border lines, key security areas, oil and gas pipelines, and other scenes [1-5]. By extracting intrusion signals in the buried optical fiber, the system realizes long-distance, wide-range intrusion detection, and real-time pre-warning. It is an effective way to monitor illegal entry, ensure communication safety, and prevent to damage pipelines. The optical fiber intrusion signal processing can be divided into two steps, namely detection and recognition. The detection function is used to locate the intrusion. According to the detection results, we can extract the intrusion signal segment to identify the type of intrusion. Therefore, accurate detection of intrusion signal is an important prerequisite for the effective operation of OFPS.

Currently, phase-sensitive optical time domain reflectometer $(\Phi-O T D R)$ is a typical structure in OFPS [6]. It can be used to detect concurrent intrusions with every small resolution cell. Fixed threshold detection is adopted in $\Phi-$ OTDR initially,

Received: 29 December 2016 / Revised: 10 March 2017

(C) The Author(s) 2017. This article is published with open access at Springerlink.com

DOI: $10.1007 / \mathrm{s} 13320-017-0399-\mathrm{z}$

Article type: Regular 
but its performance degrades dramatically in complicated application environment [7-11]. Furthermore, the constant false alarm rate (CFAR) methods are introduced to detect the intrusion signal of optic fiber [12-16]. CFAR improves the performance of OFPS in a certain extent because adaptive threshold of it, but there is still a mass of false alarms in detection results. A large quantity of false alarms will increase workload and reduce security personnel's work efficiency. To solve this problem, it is necessary to develop new algorithms to further decrease false alarms.

The proposed $\Phi$-OTDR based on detection algorithm will improve the performance of traditional CFAR. At present, most CFAR algorithms used in OFPS are based on spatial characteristics of signals. They detect the amplitude change of synthesis of two orthogonal polarization signals. However, in previous researches, characteristics of these two signals and their relationships are neglected. According to the theory of birefringence of single-mode fiber [17-21], the signals collected by OFPS are analyzed in the paper. The results show that the difference between false alarm and intrusion is obvious and robust, thus it can be used to decrease false alarms. Moreover, by analyzing and discriminating false alarm signals and intrusion signals collected in practical applications, we find that false alarm signals and intrusion signals can be distinguished from the correlation coefficient of the two orthogonal polarization signals. According to this conclusion, this paper presents a harmful intrusion detection algorithm based on correlation of orthogonal polarization signals. Further experiments prove the feasibility and effectiveness of this algorithm. In the end, the OFPS is tested in Shangweidian Village of Mentougou District in Beijing. Experiments of multiple intrusions detection are implemented and the results show the proposed algorithm can significantly decrease false alarms compared with previous methods.
The remainder of this paper is organized as follows. Section 2 gives an introduction of the proposed method. Section 3 is devoted to the correlation analysis of optical fiber signals based on birefringence, including intrusion signals, noise, and false alarm signals. The OFPS detection algorithm based on correlation of orthogonal polarization signals is proposed in Section 4. In Section 5, experiments on real data demonstrate the effectiveness of the proposed method. The discussions and conclusions are provided in Section 6.

\section{Analysis on optical fiber signals}

In this section, we introduce birefringence of the single mode optical fiber first, then analyze three types of optical fiber signals to investigate the principle of the proposed algorithm.

\subsection{Birefringence of single mode optical fiber}

Single mode optical fiber is used in OFPS to extract intrusion signals. The principle is that external stresses can lead to birefringence through the photo elastic effect. A bent fiber is shown in Fig. 1. As seen, there are two orthogonal polarization modes $\mathrm{HE}_{11}^{X}$ and $\mathrm{HE}_{11}^{Y}$. Birefringence is induced, when propagation constant $U_{x}$ and $U_{y}$ of polarization modes are not equal.

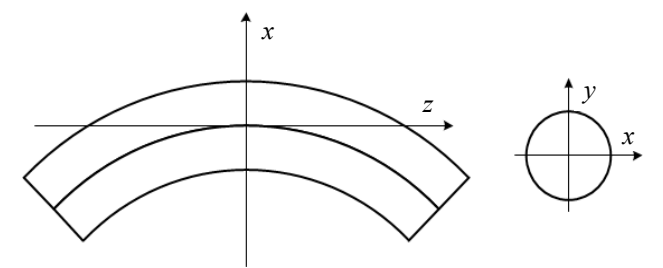

Fig. 1 Geometry of a bent fiber.

On the one hand, wavelengths of $\mathrm{HE}_{11}^{X}$ and $\mathrm{HE}_{11}^{Y}$ are equivalent when there is no bending caused by external stresses. In this situation, $U_{x}=U_{y}$, and polarization modes degenerate. Therefore, components of signal remain equal in the directions of $x$ and $y$ because of the same phase. On the other hand, the wavelengths of $\mathrm{HE}_{11}^{X}$ and $\mathrm{HE}_{11}^{Y}$ are no longer equal anymore because reflective indexes of $x$ and $y$ change differently when optical fiber is bent 
in direction of $x$ axis as shown in Fig. 1. In this case $U_{x} \neq U_{y}$, and the two polarization modes do not degenerate. Accordingly, the components in $x$ and $y$ are unequal.

Actually, direction of external stresses is rarely identical to vector sum direction of two polarization modes composition, so changes of $U_{x}$ and $U_{y}$ are unequal. Hence, amplitude fluctuation induced by intrusion is obvious in one of orthogonal polarization signals and dim in the other.

\subsection{Correlation analysis on optical fiber signals based on birefringence}

This section analyzes three kinds of typical optical fiber signals, including intrusion signals, noises, and false alarm signals.

\subsubsection{Correlation analysis on intrusion signals}

Signal I and signal $Q$ are two orthogonal polarized components of intrusion signals. They are depicted in Fig. 2. A 1-s long fragment of the signals marked with a rectangle are shown in Fig. 3. This kind of signal is detected as intrusion by CFAR. Obviously, waveform in Fig. 2(a) is significantly different from that in Fig. 2(b) which contains lots of peaks and valleys. The propagation constants of two polarization modes are distinct according to Section 2.1.
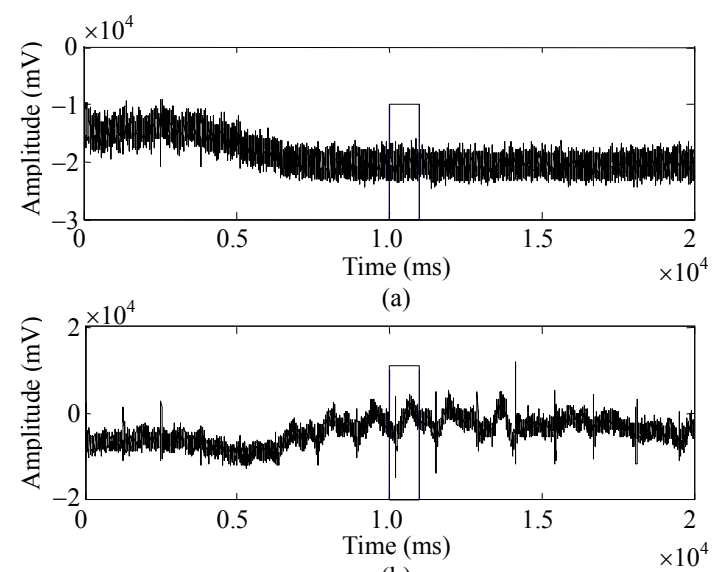

(b)

Fig. 2 Intrusion signals: (a) signal I and (b) signal Q.

In order to compare the correlation difference of three kinds of signals, following steps are taken:
(1) The signal is divided into 20 segments with each length of $1 \mathrm{~s}$.

(2) The correlation coefficients of 20 groups of two signals are calculated.

(3) Fit curve of distribution function by making statistical analysis on the correlation coefficients.

(4) The mean and variance is computed based on the curve obtained in Step (3).
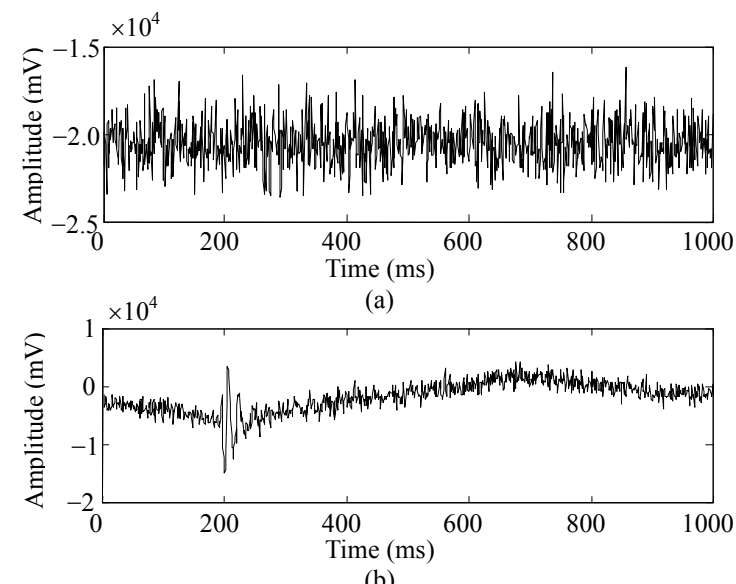

(b)

Fig. 3 Intrusion signals: (a) signal I and (b) signal Q.

The result of relevance analysis of intrusion signal is shown in Table1.

Table 1: Statistic on intrusion signals.

\begin{tabular}{ccc}
\hline Signal type & Mean & Variance \\
\hline Intrusion & 0.1662 & 0.0140 \\
\hline
\end{tabular}

The correlation coefficient is Gaussian distributed. Its mean and variance are 0.1662 and 0.140 , respectively.

\subsubsection{Correlation analysis on noise}

The noise is collected by OFPS when no external stresses are applied on optical fiber, and it cannot be detected by CFAR. Noise is shown in Fig. 4. Signal I and signal Q are two orthogonal polarized components, respectively. Segments of the signals marked by rectangle are shown in Fig. 5 . There is no prominent peak in the wavelet, which means no intrusion.

Noise signals are analyzed in the same way as in Section 2.2.1. The result is shown in Table 2. The correlation coefficient follows Gaussian distribution, 
and its mean and variance of correlation coefficient is 0.2710 and 0.0111 .

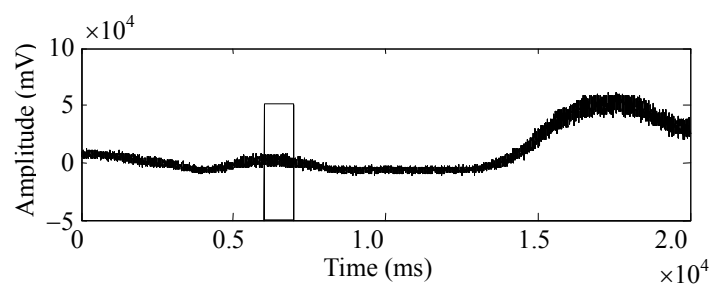

(a)

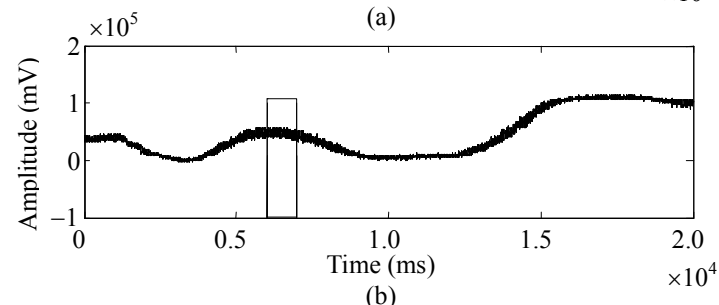

Fig. 4 Noise signals: (a) signal I and (b) signal Q.

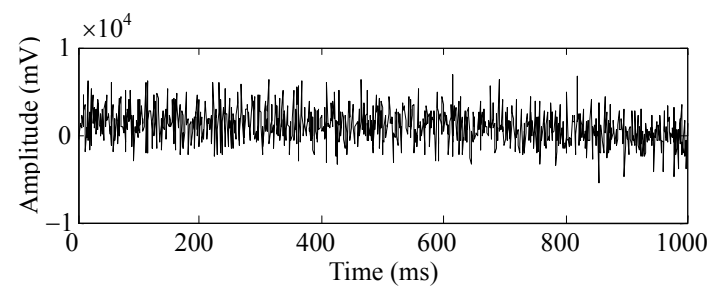

(a)

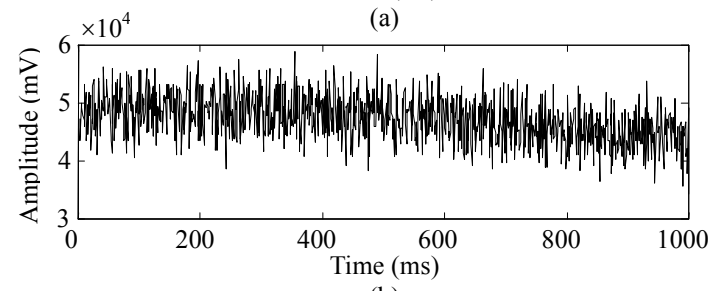

(b)

Fig. 5 Noise signals: (a) signal I and (b) signal Q.

Table 2: Statistic on noise.

\begin{tabular}{ccc}
\hline Signal type & Mean & Variance \\
\hline Noise & 0.2710 & 0.0111 \\
\hline
\end{tabular}

\subsubsection{Correlation analysis on false alarm signals}

Signal I and signal Q, orthogonal polarized components of false alarm signal, are shown in Fig. 6. Segments marked by rectangle are shown in Fig. 7. Peaks are found in both orthogonal polarization signals, and can be detected by CFAR. According to birefringence, bending induced by external stresses will lead to change in the one of orthogonal polarization signals, but the changes are synchronized and similar to false alarm signals in figures. Consequently, it is not the strain that leads to fluctuation in the false alarm signals. Statistics on alarm signals are shown in Table 3.
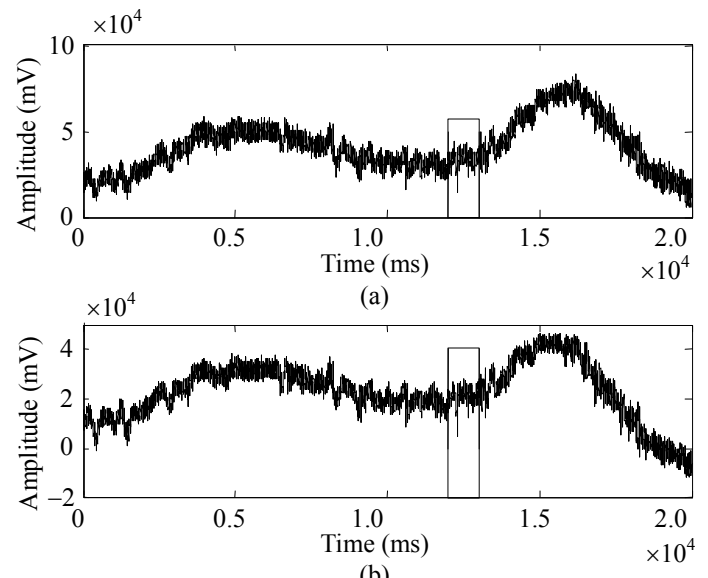

(b)

Fig. 6 False alarm signals: (a) signal I and (b) signal Q.

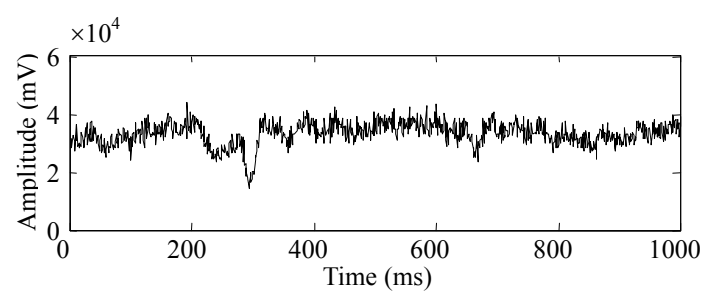

(a)

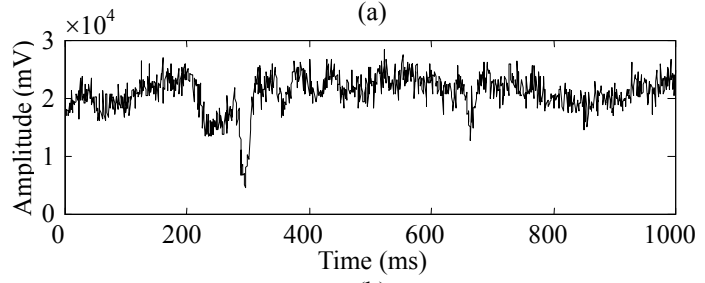

(b)

Fig. 7 False alarm signals: (a) signal I and (b) signal Q.

Table 3: Statistic on false alarm signals.

\begin{tabular}{ccc}
\hline Signal type & Mean & Variance \\
\hline False alarm & 0.7705 & 0.0163 \\
\hline
\end{tabular}

Correlation coefficient mean of false alarms is 0.7705 , and it is much greater than that of intrusion signals 0.1662 and that of noise 0.2710 . Based on these analyses, the correlation coefficient of orthogonal polarization signals is an effective feature to distinguish intrusion and false alarm, and it can be used to exclude false alarms in the result obtained by CFAR.

\section{Detection algorithm based on correlation of cross-polarization signals}

According to the correlation analysis, this paper proposes a harmful intrusion detection algorithm based on correlation of orthogonal polarization signals. The main procedures of the proposed method are shown in Fig. 8. 


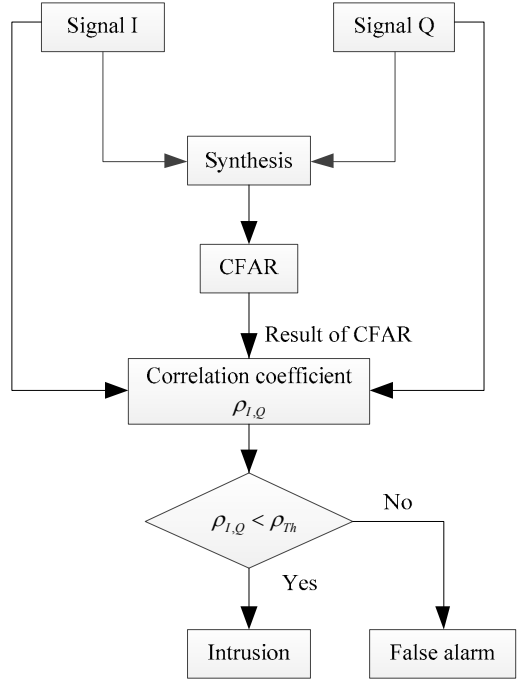

Fig. 8 Proposed algorithm.

(1) Synthesize signal $I$ and signal $Q$ collected by OFPS into $D$ as (1):

$$
D=I^{2}+Q^{2}
$$

(2) Apply CFAR to detect $D$ to extract alarm signals.

(3) Calculate correlation coefficient between $I$ and $Q$ of alarm signal as (2), and compare it with presupposed threshold.

$$
\rho_{I, Q}=\operatorname{Cov}(I, Q) / \sqrt{D(I) \cdot D(Q)}
$$

where $\rho_{I, Q}$ is correlation coefficient, $\operatorname{Cov}(I, Q)$ is covariance of $I$ and $Q$, and $D(I)$ and $D(Q)$ are variance of $I$ and $Q$, respectively. If $\rho_{I, Q}$ is less than $\rho_{\text {Th }}$ which is the threshold, the alarm is judged as an intrusion otherwise it is a false alarm.

Hypothesis testing is used to determine the threshold. Firstly, all sample of false alarm signals are divided into segments of $1 \mathrm{~s}$. Subsequently, their $\rho_{I, Q}$ of them are calculated. Then, fit all $\rho_{I, Q}$ values into Gaussian distribution. Last, calculate $\rho_{\text {Th }}$ of hypothesis testing from probability distribution function based on false alarm rate which is set as $1 \times 10^{-7}$.

\section{Experiment and analysis}

\subsection{Collection of real data}

In order to validate correlation feature of optical fiber signals and performance of proposed algorithm, test data is collected in Shangweidian Village of
Mentougou District in Beijing. A 1000-m long optical fiber is buried $20 \mathrm{~cm}$ under the ground.

Signals are collected under three conditions as follows.

(1) Experiment site without any external intrusion interference.

(2) Testers jog at the $700 \mathrm{~m}$ to the end of optical fiber for $8 \mathrm{~s}$.

(3) Testers dig the ground with a pickaxe at the $500 \mathrm{~m}$ to the end of optical fiber for $8 \mathrm{~s}$.

\subsection{Experiment to verify correlation feature of optical fiber signals}

The signals collected by OFPS are analyzed in Section 2.2. Alarms in Condition (1) are considered as false alarms. In addition, alarms in non-intrusion positions in Conditions (2) and (3) are false alarms too. The results are shown in Table 4.

Table 4: Analysis on four types of measurements.

\begin{tabular}{ccccc}
\hline & Noise & Jogging & Digging & $\begin{array}{c}\text { False } \\
\text { alarm }\end{array}$ \\
\hline $\begin{array}{c}\text { Mean of correlation } \\
\text { coefficient }\end{array}$ & 0.2588 & 0.1714 & 0.1594 & 0.7493 \\
$\begin{array}{c}\text { Variance of correlation } \\
\text { coefficient }\end{array}$ & 0.0133 & 0.0160 & 0.0122 & 0.0194 \\
\hline
\end{tabular}

Accordingly, the correlation coefficient of the false alarm signal is much larger than two intrusion signals. This result agrees with analysis on signals collected by OFPS in Section 2.2. It proves that the correlation coefficient of the false alarm is greater than that of the intrusion signals. And this is a general characteristic in OFPS.

\subsection{Experiments of the OFPS detection algorithm}

Both CFAR and the proposed algorithm are used to process signals under three situations, including non-intrusion, jogging, and digging. Then, the performance of these two algorithms will be compared in detection probability and false alarm probability.

The detection results of signals without intrusion are shown in Figs. 9 and 10. Horizontal axis and vertical axis are situation and time, respectively. According to Fig. 9, it is clear that there are a lot of false alarms in the position of $300 \mathrm{~m}$ and other sporadic false alarms after CFAR detection. The 
results of the proposed algorithm are displayed in Fig. 10. Apparently, most false alarms are eliminated effectively.

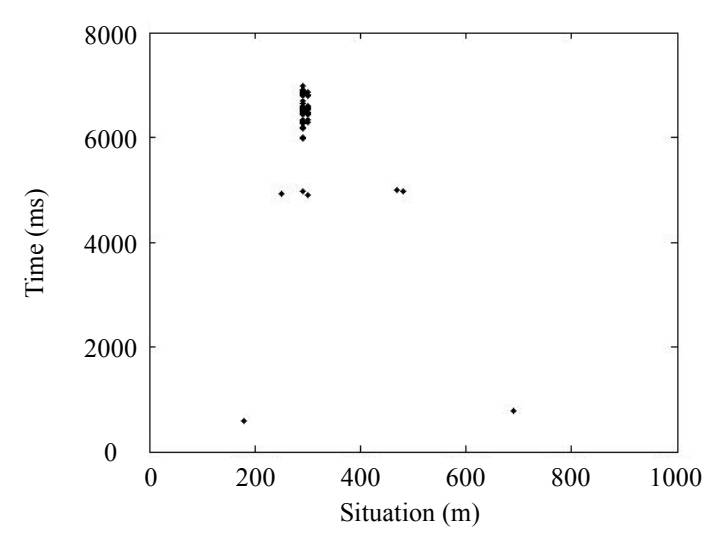

Fig. 9 Detection result of CFAR.

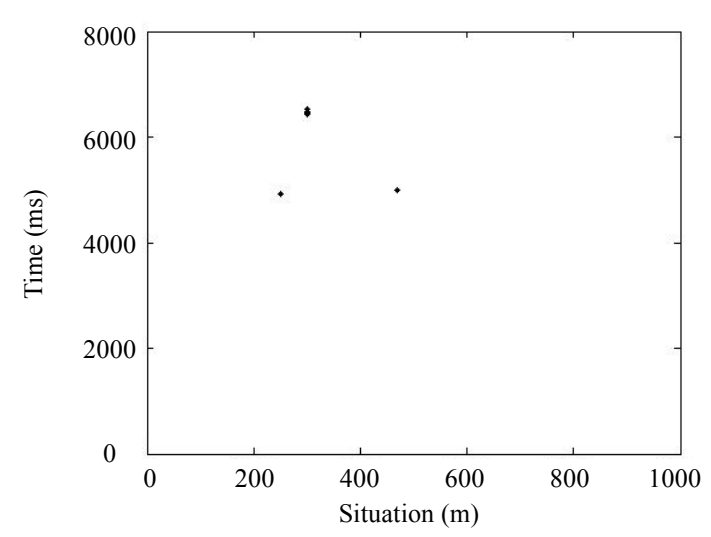

Fig. 10 Detection result of proposed method.

According to the detection outcome of jogging shown in Figs. 11 and 12, the proposed algorithm not only eliminates false alarm, but also detects intrusion in position of the $700 \mathrm{~m}$ to the end, which coincides with the experiment setup.

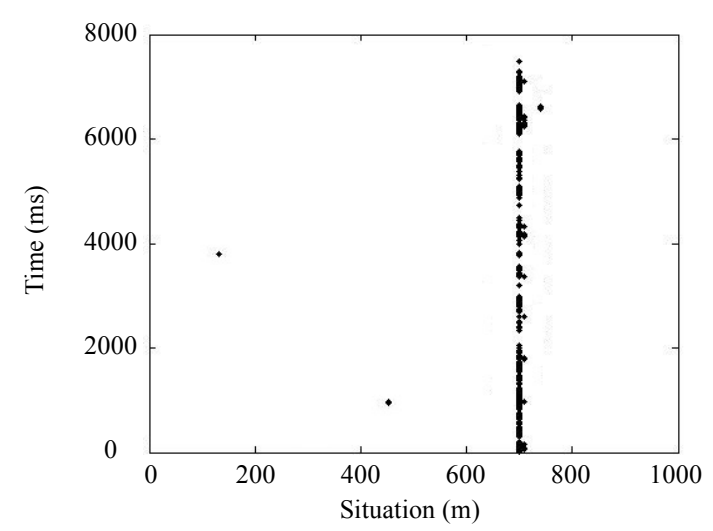

Fig. 11 Detection result of CFAR.

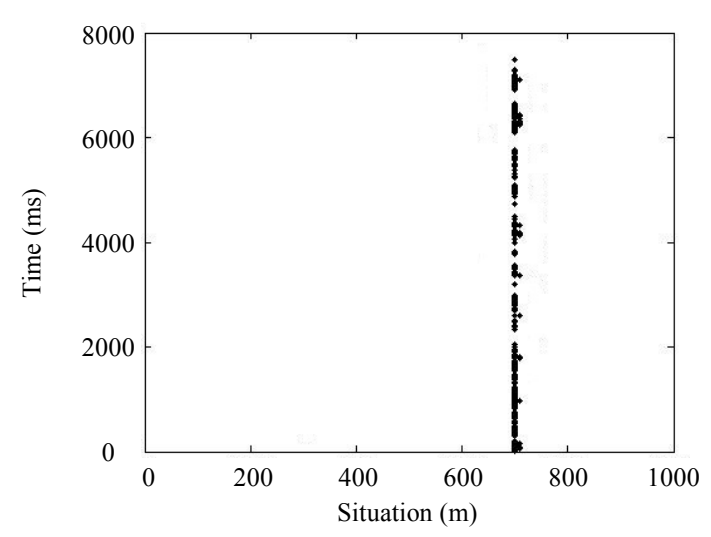

Fig. 12 Detection result of proposed method.

Figures. 13 and 14 show the detection results of the digging signal. As seen in the figure, intrusion at position of $500 \mathrm{~m}$ is effectively detected, and the duration of the alarm exactly agrees with the experimental setup. Significantly, false alarms in Fig. 13 are noticeably less than those in Fig. 14, so the proposed algorithm achieves better performance in reducing false alarms.

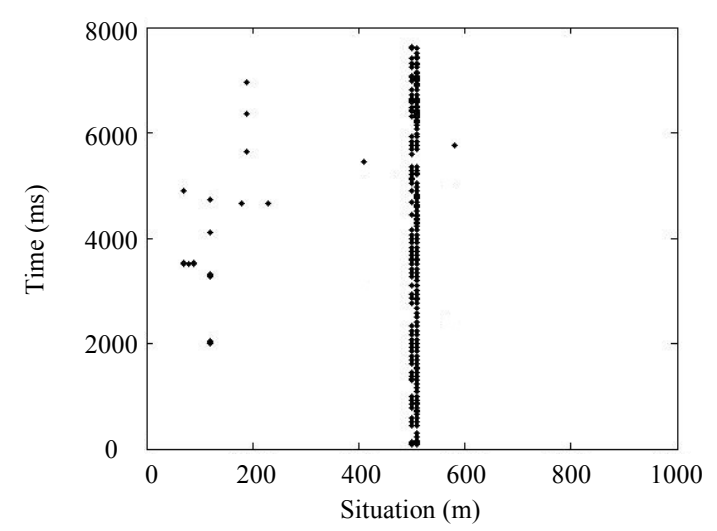

Fig. 13 Detection result of CFAR.

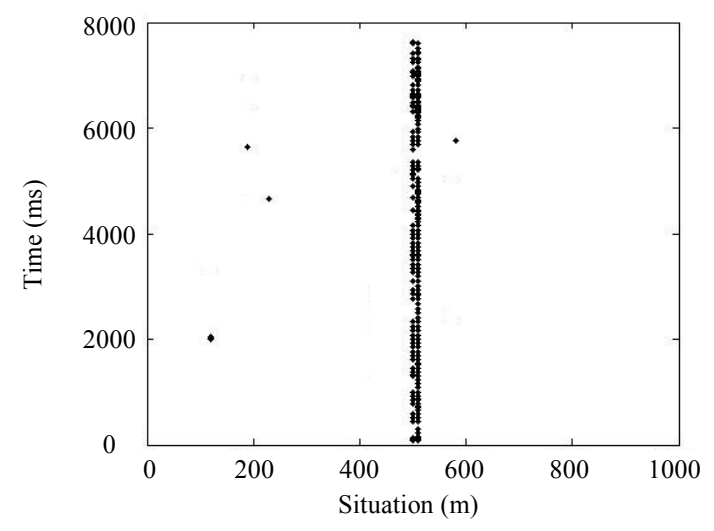

Fig. 14 Detection result of proposed method. 
In order to demonstrate the availability of proposed algorithm, we count the number of both false alarms and alarms at the intrusion site. The results are given in Tables 5 and 6 . As can be seen, the proposed algorithm performs better than CFAR. The detection performance of both methods is comparable, but the proposed algorithm reduces false alarms by $87.8 \%$.

\begin{tabular}{ccc} 
Table 5: Number of false alarms. & \\
\hline Type of signal & CFAR & Proposed method \\
\hline No intrusion & 137 & 10 \\
Jogging & 3 & 0 \\
Digging & 65 & 15 \\
\hline
\end{tabular}

Table 6: Number of alarms in intrusion site.

\begin{tabular}{ccc}
\hline Type of signal & CFAR & Proposed method \\
\hline Jogging & 1246 & 1246 \\
Digging & 471 & 471 \\
\hline
\end{tabular}

By comparing detection results of three types of signals, it is clear that the proposed OFPS detection algorithm can remove the harmless interferences based on CFAR detection results without the degradation of the detection performance.

\section{Discussions and conclusions}

In this paper, false alarm in OFPS is studied. Based on the theory of birefringence of single mode optical fiber, we find that the correlation coefficient of two orthogonal polarization signals can be used to distinguish false alarm and intrusion. According to the results of statistical analysis on correlation coefficient of intrusion signals, noises, and false alarm signals, we find that correlation coefficient of false alarms signals is notably greater than that of intrusion signals. In addition, we show that the difference in correlation feature of optical signals commonly exists in OFPS.

We propose a harmful intrusion detection algorithm based on the correlation analysis of orthogonal polarization signals. Firstly, the synthesis of two cross-polarization signals is detected by CFAR to get alarms. Afterwards, the coefficient of correlation of alarm signals is calculated. Eventually, once the coefficient is lower than the presupposed threshold, the alarm is judged as an intrusion, or it is a false alarm.

To test the algorithm, we design experiments on real data which is collected in Shangweidian Village of Mentougou District in Beijing. The threshold is determined through hypothesis testing from probability distribution function and false alarm rate is set as $1 \times 10^{-7}$. The results show that the proposed algorithm outperforms CFAR in terms of false alarms. Accuracy of OFPS can be significantly improved because false alarms are drastically reduced and intrusions are detected accurately as well.

\section{Acknowledgement}

This work was supported by the National Nature Science Foundation of China (Grant Nos. 61571014, 61601006); Beijing Nature Science Foundation (Grant No. 4172017); General project of science and technology program of Beijing Education Commission(Grant No. KM201610009004).

Open Access This article is distributed under the terms of the Creative Commons Attribution 4.0 International License (http://creativecommons.org/ licenses/by/4.0/), which permits unrestricted use, distribution, and reproduction in any medium, provided you give appropriate credit to the original author(s) and the source, provide a link to the Creative Commons license, and indicate if changes were made.

\section{Reference}

[1] Z. G. Qu, H. Feng, Z. M. Zeng, J. C. Zhuge, and S. J. Jin, "A SVM-based pipeline leakage detection and pre-warning system," Measurement, 2010, 43(4): 513-519.

[2] J. Kang and Z. H. Zou, "Time prediction model for pipeline leakage based on grey relational analysis," Physics Procedia, 2010, 25(2): 2019-2024.

[3] W. Liang, L. L. Lu, and L. B. Zhang, "Coupling relations and early-warning for 'equipment chain' in long-distance pipeline," Mechanical Systems and Signal Processing, 2013, 41(1-2): 335-347.

[4] W. Liang, L. B. Zhang, Q. Q. Xu, and C. Y. Yan, “Gas pipeline leakage detection based on acoustic technology," Engineering Failure Analysis, 2013, 31(6): 1-7. 
[5] T. T. Zhang, Y. F. Tan, H. X. Yang, J. H. Zhao, and X. D. Zhang, "Locating gas pipeline leakage based on stimulus-response method," Energy Procedia, 2014, 61: 207-210.

[6] Q. Y. Lv, L. J. Li, H. B. Wang, Q. Li, and X. Zhong, "Influences of laser on fiber-optical distributed disturbance sensor based on $\Phi-O T D R$," Infrared and Laser Engineering, 2014, 43(12): 3918-3923.

[7] H. F. Martins, S. Martin-Lopez, P. Corredera, M. L. Filograno, O. Frazão, and M. Gonzáez-Herraez, "Coherent noise reduction in high visibility phase-sensitive optical time domain reflectometer for distributed sensing of ultrasonic waves," Journal of Lightwave Technology, 2013, 31(23): 3631-3637.

[8] Q. Li, C. X. Zhang, L. J. Li, and X. Zhong, "Localization mechanisms and location methods of the disturbance sensor based on phase-sensitive OTDR," Optik-International Journal for Light and Electron Optics, 2014, 125(9): 2099-2103.

[9] Q. Lin, C. X. Zhang, and C. S. Li, "Fiber-optic distributed sensor based on phase-sensitive OTDR and wavelet packet transform for multiple disturbances location," Optik-International Journal for Light and Electron Optics, 2014, 125(24): 7235-7238.

[10] A. R. Bahrampour and F. Maaoumi, "Resolution enhancement in long pulse OTDR for application in structural health monitoring," Optical Fiber Technology, 2010, 16(4): 240-249.

[11] L. D. Lu, Y. J. Song, X. J. Song, X. P. Zhang, and F. Zhu, "Frequency decision multiplexing OTDR with fast signal processing", Optics \& Laser Technology, 2012, 44(7): 2206-2209.

[12] H. Q. Qu, T. Zheng, F. K. Bi, and L. P. Pang, "Vibration detection method for optical fiber pre-warning system,” IET Signal Process, 2016, 10(6): 692-698.

[13] H. Q. Qu, T. Zheng, L. P. Pang, and X. L. Li, “A new two-dimension method to detect harmful intrusion vibrations for optical fiber pre-warning system," Optik-International Journal for Light and Electron Optics, 2016, 127(10): 4461-4469.

[14] R. L. Zhang, W. X. Sheng, and X. F. Ma, "Improved switching CFAR detector for non-homogeneous environments," Signal Processing, 2013, 93(1): $35-48$

[15] G. V. Weinberg, "Management of interference in Pareto CFAR processes using adaptive test cell analysis," Signal Processing, 2014, 104(104): 264-273.

[16] B. Shi, C. P. Hao, C. H. Hou, X. C. Ma, and C. Y. Peng, "Parametric Rao test for multichannel adaptive detection of range-spread target in partially homogeneous environments," Signal Processing, 2015, 108(108): 421-429.

[17] M. Karimi, T. Sun, and K. T. V. Grattan, "Design evaluation of a high birefringence single mode optical fiber-based sensor for lateral pressure monitoring applications," IEEE Sensors Journal, 2013, 13(11): 4459-4464.

[18] L. Palmieri, A. Galtarossa, and T. Geisler, "Distributed characterization of bending effects on the birefringence of single-mode optical fibers," Optics Letters, 2010, 35(14): 2481-2483.

[19] Z. Y. Li, C. Q. Wu, H. Dong, P. Shum, C. Y. Tian, and S. Zhao, "Stress distribution and induced birefringence analysis for pressure vector sensing based on single mode fibers," Optics Express, 2008, 16(6): 3955-3960.

[20] B. Y. Kim and S. S. Choi, "Backscattering measurement of bending-induced birefringence in single mode fibres," Electronics Letters, 1981, 17(5): 193-194.

[21] O. G. Leminger, "Stress birefringence in single-mode fibres," Electronics Letters, 1977, 13(12): 370-371. 